\title{
20
}

\section{Towards Active Management Information}

\section{Systems}

\author{
P. Mertens, J. Hagedorn, M. Fischer, N. Bissantz, and M. Haase
}

Bavarian Research Center for Knowledge-Based Systems (FORWISS), Am Weichselgarten 7, 91058 Erlangen-Tennenlohe, Germany, Tel.: ++49-911-5302-264, Fax.: ++49-911-536634, E-mail:mertens@wiso.uni-erlangen.de

\begin{abstract}
With Active Management Information Systems (Active MIS) we delegate more responsibility to the system. Different types of Active MIS are arranged in a framework that is defined by two scales. We focus on one of the scales, namely the intensity of user interaction, which also serves as the guideline of the paper. At the same time we give examples to illustrate how Active MIS can contribute to the solution of a user's problem.
\end{abstract}

\section{Keywords}

Active Management Information Systems, Data Mining, Critiquing, Navigation, AddresseeOrientiation, Automatic Reporting

\section{INTRODUCTION}

Computer-assisted management information originally meant printing masses of paper ("number cemeteries"). Analyzing the sales department of a company producing ballbearings we found that each manager received an average of 23 reports a day. Their Management Information Systems (MIS) were totally system-driven.

Meanwhile we have mastered dialogue, hypertext, and hypermedia systems. Vendors of EIS offer the features shown in Figure 1 (left). From their customers they expect the characteristics listed on the right hand side which seems to be far from reality, at least as far as top managers are concerned. 


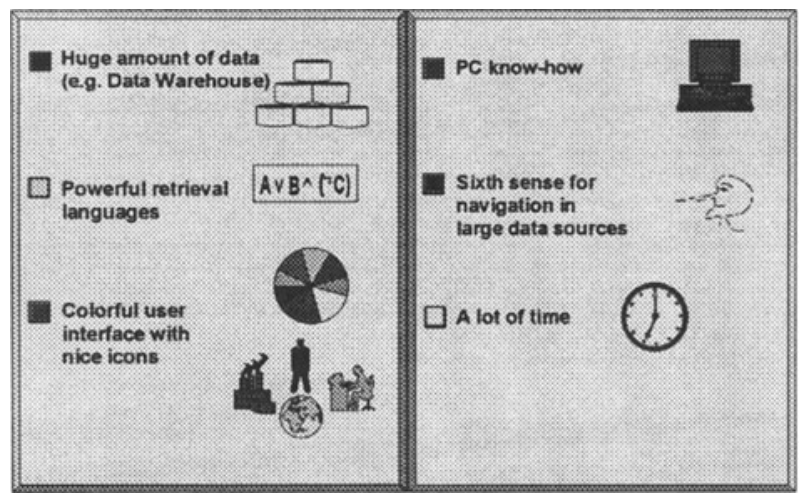

Figure 1 Features and requirements of MIS software

This kind of MIS is fully user-controlled and often result in very time-consuming dialogues, e.g. with a lot of windows opened, menu-entries selected, hotwords clicked, etc., and finally the user might end up being "lost in space".

For that reason we need another paradigm called Active MIS which means that we delegate more responsibility to the system. The ultimate goal is to get reports where

- the selection of critical information from huge databases and data warehouses is the task of the system, not that of the manager,

- the presentation of this information is automated as well, and

- the presentation style considers the decision tasks and the personal preferences of the addressee.

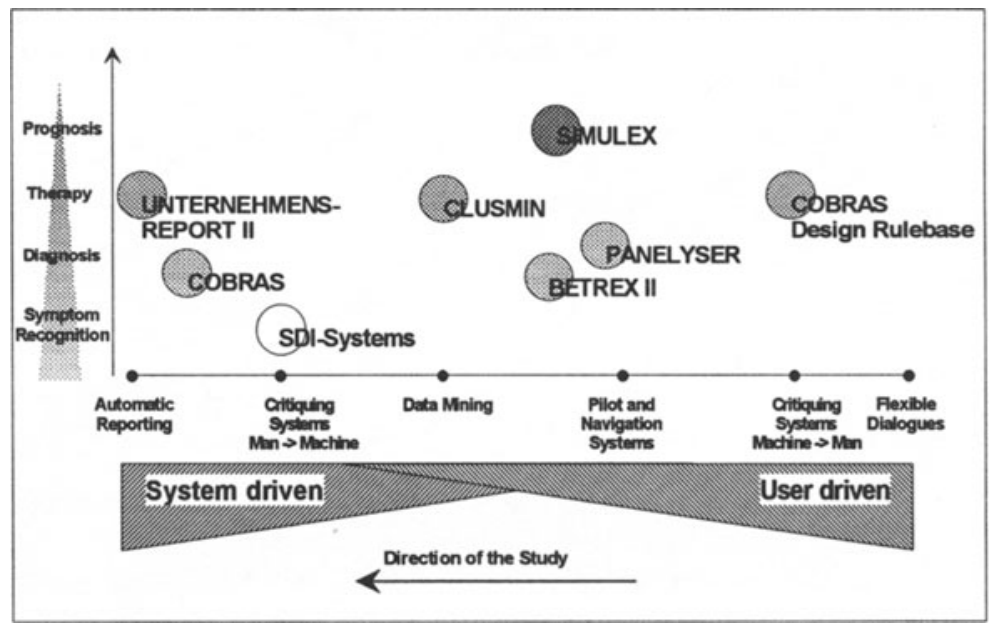

Figure 2 Overview 
Figure 2 shows the structure of this paper. Starting from the state of the art, which is interactive MIS, at the right end of the scale we will go back step by step to the goal on the left hand side. We will pass several "stations" which at the same time mark the chapters of our paper. Some innovative examples of Active MIS (prototypes and running systems) that we have developed with German companies from several industries will be outlined. Moreover we will try to overcome techniques where only facts are presented in order to diagnose weaknesses: we want to progress to a stage where we can give therapy suggestions, and then even forecast the impact of the means chosen. Some hints to actual research tasks ("white spots on the map") are given.

\section{CRITIQUING SYSTEMS (MACHINE $>$ MAN)}

Critiquing Systems offer many degrees of freedom to the user. They only interact when a mistake occurs. They can be compared to an observer with special domain knowledge who is able to give a decisive hint at the right time.

The example we describe below was developed in cooperation with the Bavarian Research Center for Knowledge-Based Systems (FORWISS) and GfK AG, Nuremberg, the leading German market research institute.

Besides other kind of research results the institute provides retail audit data to track the market development, especially the analysis of time series, such as the sales volume of a product line in varying distribution channels. After the comprehensive survey of the data, the latest research results must be presented to the clients of the institute in a few days. Therefore, more automation is welcomed.

\section{System Description: COBRAS}

There is a new system now, called COBRAS (Client Oriented Branch Reporting and Analysis System), that supports the market researcher making presentation slides. It simulates the market researcher's working methods and provides know-how about many different analysis types, presentation structures, the design of graphical representation, the preferences of user and client. With the help of that knowledge the Active MIS is able to generate a presentation automatically or interactively.

At this point we want to draw attention to the critique-component of the system that advises the user when selecting the right visualization technique.

The knowledge consists of general guidelines for business graphics, Corporate Identity recommendations and expert know-how of the market researchers. For example the following rules are part of that knowledge:
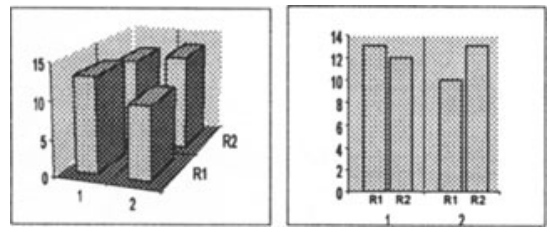

Rule example 1:

If a third axis is needed, it should be integrated in the $x$-axis using groups (Figure 3).

Figure 3 Rule example 1 


\section{Rule example 2:}

If the diagram consists of more than 16 columns, its type should be changed into a line diagram (Figure 4).

The rule base gets active as soon as a presentation chart form is filled with new data. For each form a default value is given, especially because it helps to understand the user's intention. Based on this the design component of COBRAS checks, if the default value is adequate, criticizes if necessary and takes on the task of fine formatting, such as labeling axes and data items with the appropriate letter size and position.
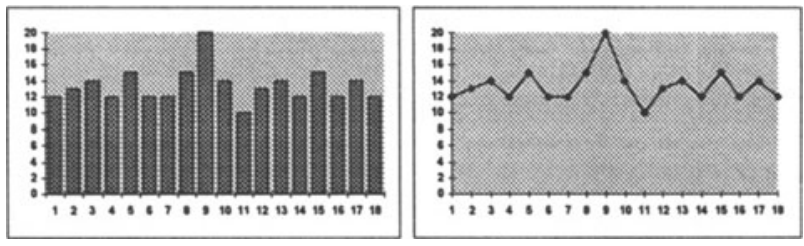

Figure 4 Rule example 2

\section{PILOT AND NAVIGATION SYSTEMS}

At the International Conference on Economics / Management and Information Technology 1992 in Tokyo Nobel prize-winner Herbert Simon was the keynote speaker. He pointed out the following aspect:

"Information is not scarce. What is scarce is manager's time to attend to all the information that is available."

It may seem trivial, but the following speakers picked up these words again and again symptom enough that there might be an existing drawback of information systems. The escape attempt to inform managers only on basis of aggregated data is dangerous because of possible compensations at low or medium aggregation levels. But these have to be recognized. Therefore, we'd like to quote Simon again:

"Information systems are best viewed not as providers of information, but as filters for information."

What we really need are systems which support us to filter the enormous data sources, help to navigate or pilot through the data floods, and to cover the information needs of the addressee. 
PANELYSER is an example for a pilot system that has been developed in cooperation with the GfK as well. This application acts as an intelligent front-end system that supports the market researcher in accessing the GfK databases and creating a management report.

\section{System Description: PANEL YSER}

Like COBRAS the system PANELYSER focuses on retail audit data, but also considers data about promotion activities. A multitude of retail outlets supplies the market research institute with information concerning sales, purchases, stock levels, prices, and promotions. The extrapolated data provide more concise information through different aggregation levels and through calculated ratios such as market share or numerical distribution. Therefore, the analyzer is able to run detailed analyses of the market "success" of special product variations in different market segments.

The knowledge of a product group's structure that is necessary to aggregate the retail audit data is stored implicitly in the database. PANELYSER analyzes product group by product group (e.g. Color TV). The short-term analysis always deals with the current period like February/March 1996 in comparison to the corresponding period of the year before. In contrast, the long-term analysis usually handles periods of the last four years.

The whole application combines an expert system for the data analysis with a graphical user interface.

By a top-down analysis, PANELYSER attempts to locate the most interesting market segments, such as brands, retail channels, product segments, regions, etc. PANELYSER looks for the causes and compensating effects on every level of the analysis path. The central focus of the market analysis is based on the absolute change of sales units. To find out the objects that are most responsible for the market development, the system applies an algorithm using the "ABC"-analysis.

Once PANELYSER has found where the market development has occurred, it continues the top down analysis (Figure 5). The system starts with objects that have influenced the examined development most and arranges them in a descending order (Step 1). Step by step the system now accumulates the values for the objects until the sum of sales value rate surpasses a threshold (Step 2). A second threshold further reduces the objects to those that are truly significant. In addition, the system selects objects with considerable relative changes of sold units, so that the market researcher's attention can be directed as well to "small" brands that enter the market (Step 3).

For every "significant" object the analysis path is continued. The result is a widely branched analysis tree that mirrors all important changes in the market from the top to a special product or even a variant of it. 


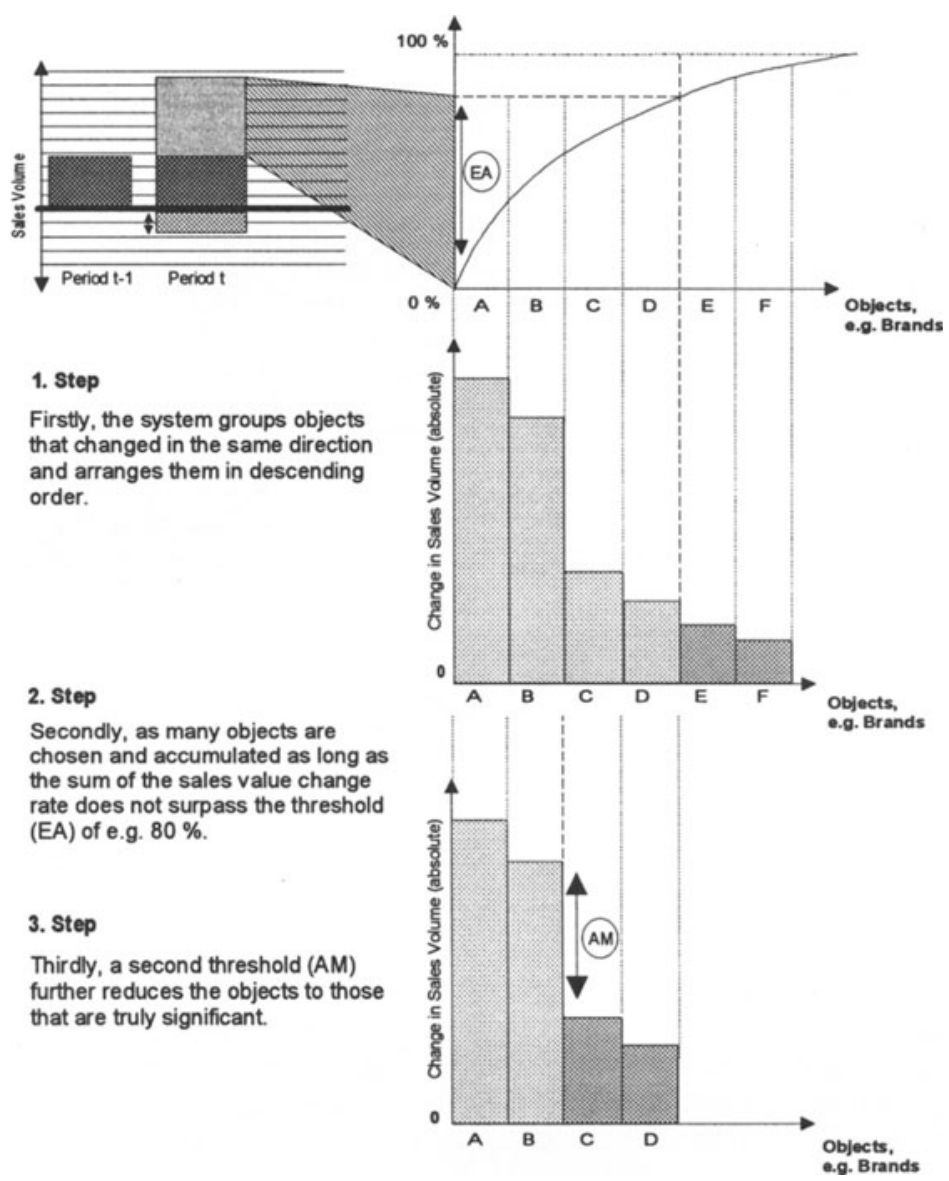

Figure 5 Significance thresholds of PANELYSER

Only the significant objects found in the short-term analysis are further examined. It is very interesting whether the development is subject to a trend or whether it happened to be a "blip". The visualization of the market share development includes hints to special events. A hyperlink ( $\triangle$-sign) draws the user's attention to promotion campaigns, etc.

The market researcher normally wants to find why sales have shifted. A database that combines retail audit and consumer panel data provides a substantial amount of information to answer those questions. For example, the reason for an increase of a product's market share can be attributed to a decrease in product price, a better distribution ratio, more promotion campaigns, or a combination of several marketing instruments. The system has to analyze whether more households buy a special product for the first time and how many households buy only that product. One method to assist the analysis is the Parfitt-Collins-algorithm (Parfitt, 1968). 
The results are presented in a verbal report, by hypertext and dynamically generated business charts.

Figure 6 shows how PANELYSER generates varied text by a lot of different components like the random text generator, the grading functionality, the product group-specific definition database, and the analysis results.

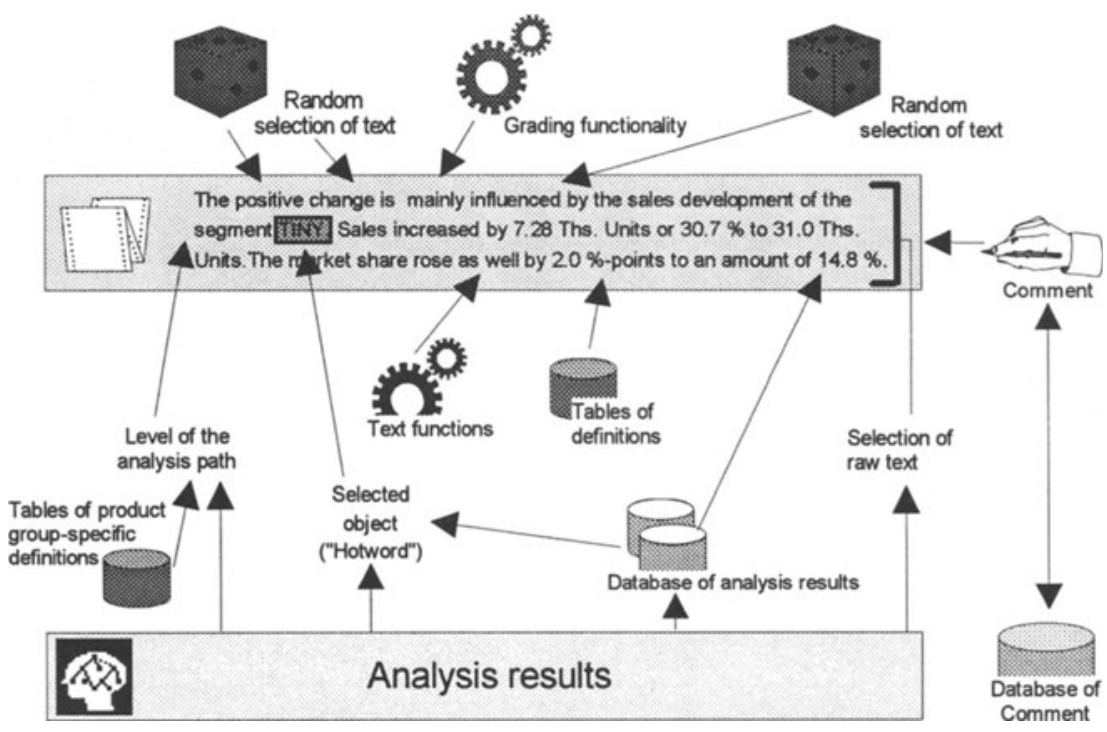

Figure 6 Text components

PANELYSER shortens the time necessary for the analysis of a product group from two or three days to a few hours.

\section{Analyzing Profitability Data}

Modern software packages for accounting and controlling provide extremely large databases that can be queried on-line and in an ad hoc manner. To facilitate data analysis, available software tools support simple querying and multi-dimensional views. Managers, however, are left alone with detecting the most interesting data constellations.

In the statement of operating results a short-term analysis on profit or loss is completed to discover profit contributions of objects, such as individual departments, customer groups or products. Imagine a company that sells three product groups in several regions to different groups of customers. While product managers for example want to know the actual sales by product in each region, they also need to compare actual sales to projected sales. As examination of performance continues, perspectives may change, and additional views are required. Figure 7 shows example reports and the multi-dimensional model they are based on. For thorough examination controllers must navigate through vast and complex hierarchies which may hide important facts deep in the data. 


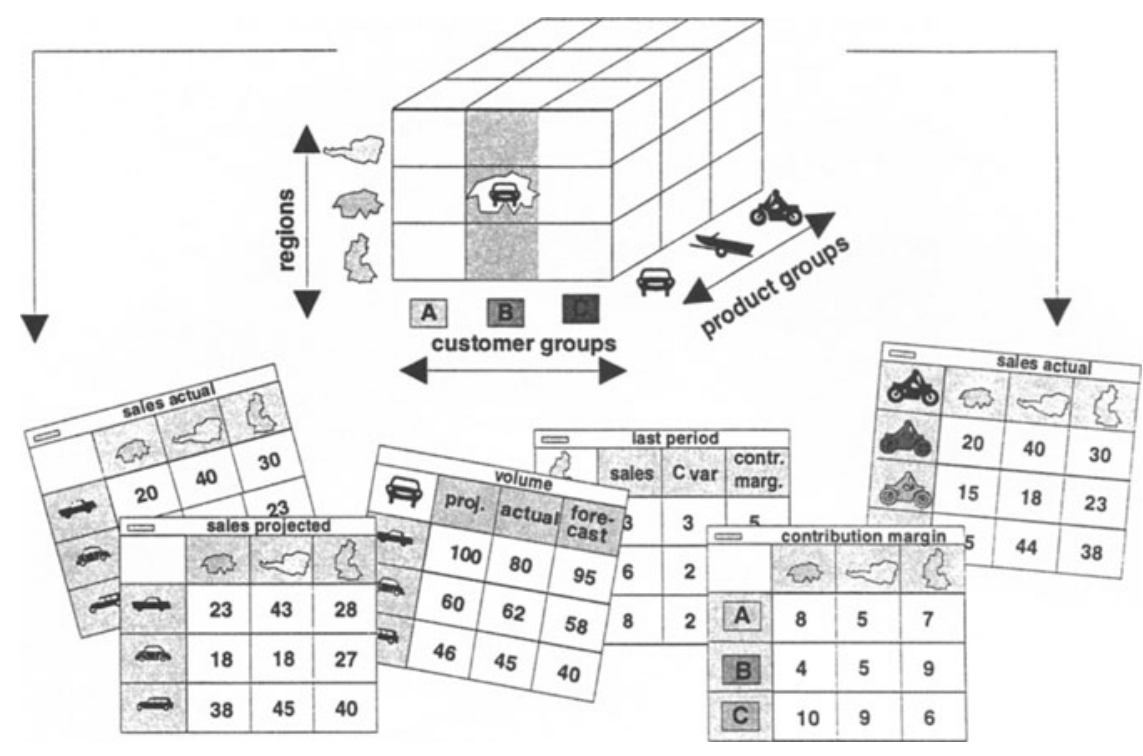

Figure 7 Operating results reports

As a consequence, sweeping changes could remain undetected for a long time. Therefore, we see two important challenges for IS research. First, tools are needed that help controllers to cope with the traditional task of finding the best way through results hierarchies. Second, algorithms may be useful that are capable of automatically extracting meaningful patterns in an unprecedented way. The latter is also known as the task of knowledge discovery, a new and promising research field that has attracted a lot of interest in recent years (Stonebraker et al., 1993).

\section{System Description: BETREX}

In the BETREX project, we incorporated algorithms attacking both problems in one system. The research was done in cooperation with the Technical University of Dresden, Germany, (Prof. Dr. W. Uhr). BETREX is designed to generate management summaries from operating results data.

The evaluation of the operating performance is based on key figures referring to objects which are described by classifying criteria. There are basic figures, such as quantities, sales, costs, computed ratios (profit contribution, sales per agent, etc.) and nominal attributes, such as region, product, or salesperson. The structure of a database is defined by the existing criteria and attribute values. Figure 8 depicts items of operating results accounting. Each item of the database represents one order item of a customer invoice, plus additional information, for instance from master data. This organization allows multi-dimensional views and reports. To give an example, a report about sales per customer group would require sorting by the criterion "customer group" and aggregating of the according figures. 


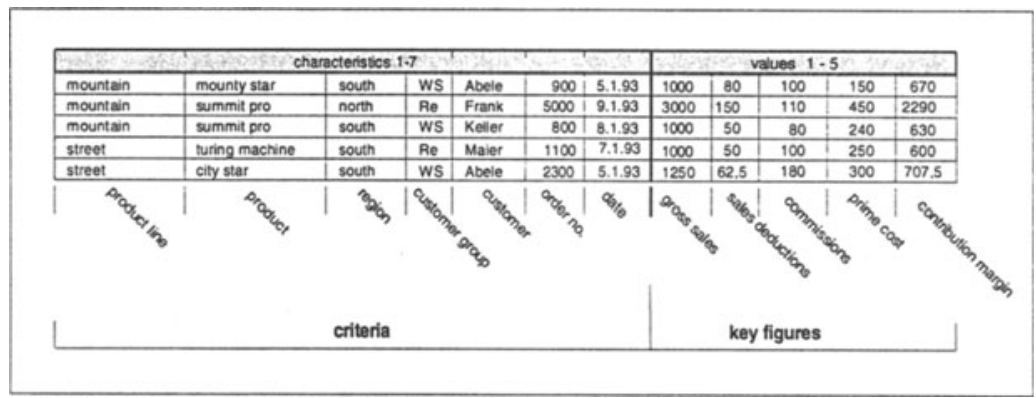

Figure 8 Operating results data

\section{Navigation Method}

The navigation filter of BETREX, as mentioned above, imitates how human controllers would try to find their path through operating results hierarchies. Analysis typically starts with some variance on a highly aggregated level and continues with examinations on different disaggregated levels.

Figure 9 depicts the mode the filter works with example data of a company selling bicycles.

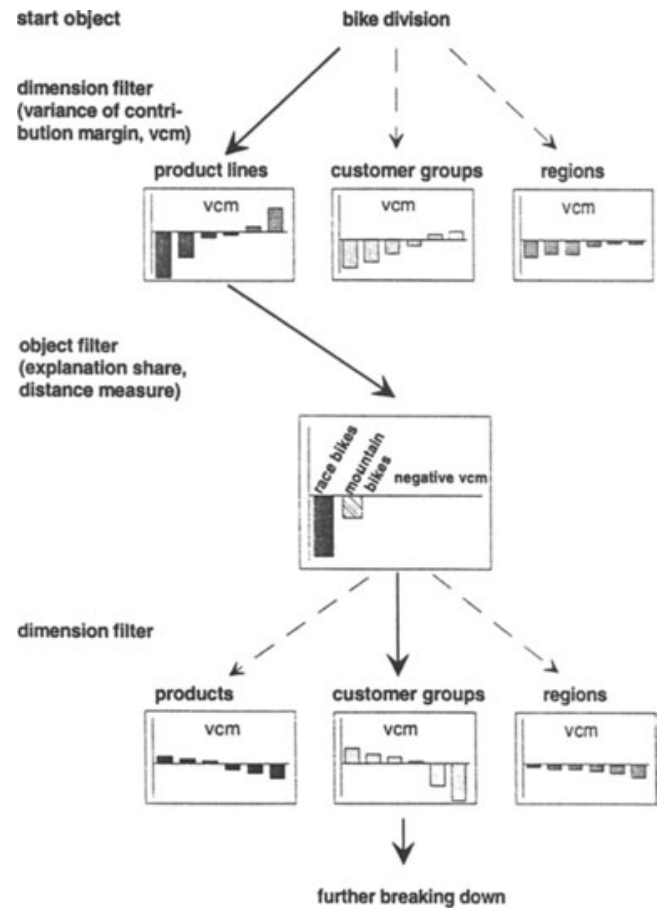

Figure 9 Navigation filter 
First, the controller chooses a start object. If none is selected, the system itself selects a start object based on the report addressee's area of responsibility. The objects which are the main cause for the out-of-line situation can be hidden in every dimension available. Therefore, the system must decide on one of three possible hierarchies for the first step of analysis: products, customers, regions.

$$
\sigma=\sqrt{\frac{1}{n} \sum_{i=1}^{n}\left(x_{i}-\bar{x}\right)^{2}}
$$

Figure 10 Navigation measure

Every bar in the picture represents the variance of a single object in the next subordinated hierarchy, for instance product line one. Generally speaking, the fewer objects cause the variance, the more likely it is that the dimension is responsible for the out-of-line situation in business reality. Accordingly the hierarchy selection is based on a measure that is similar to statistical standard deviation and increases over the objects of every dimension with rising deviation (Figure 10).

In our example, the dimension product lines is selected as the divider. Next, we must decide on which objects the analysis is to be continued. This is done by using a so-called object filter that works with an explanation share and a distance measure. Basically, the system traces a pre-defined explanation share of the variance (e.g. 80\%) of the higher level in each case. However, to avoid analyzing too many objects, the list is pruned when the difference between two objects falls below the threshold determined by the distance measure (e.g. $50 \%$ ). This process continues until the variance is explained sufficiently, or no further hierarchies are available.

The system analyzes both the causing and the compensating objects. Furthermore, each object on the navigation path is examined. The system detects changes in product and customer structure, important differences between objects on the same level, and effects on objects on higher levels of the hierarchy.

\section{Case Study}

We tested our algorithms with data from a pharmaceutical company for example. The data represented sales of two periods for a certain division of the company. The data volume was about 10,000 records. We will concentrate on the most interesting finding that is achieved in dialogue with the system in about 15 minutes.

The system starts with proposing to look at the dimension product groups. This proposal is made due to the deviation over the elements of this dimension. As a matter of fact, the element product group FBM explains $87 \%$ of the profit contribution deviation on the division level. (In the picture several steps of analysis are omitted for better understanding.) The system searches its way through the hierarchies and stops after four iterations telling the user that $70 \%$ of the profit contribution deviation are caused by the object customer group 10/article 99938 . 


\section{First step of top-down navigation}

The deviation of the profit contribution (1.4 Mio.) is explained best by dimension product group. $87 \%$ of the deviation are caused by product group FBM. Slight compensations were detected.

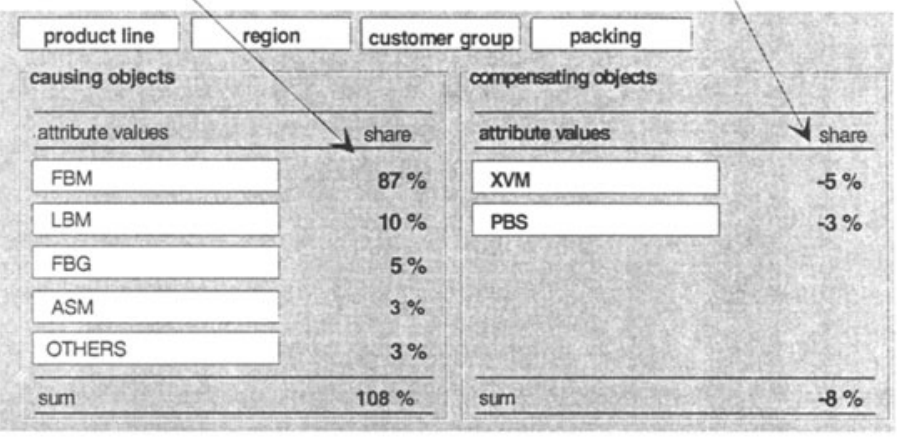

\section{Object analysis after four "drill downs"}

Object article 99938/customer group 10 explains $70 \%$ of the profit contribution deviation. Profit contribution flow analysis shows that the deviation is mostly due to variances in cost per unit.

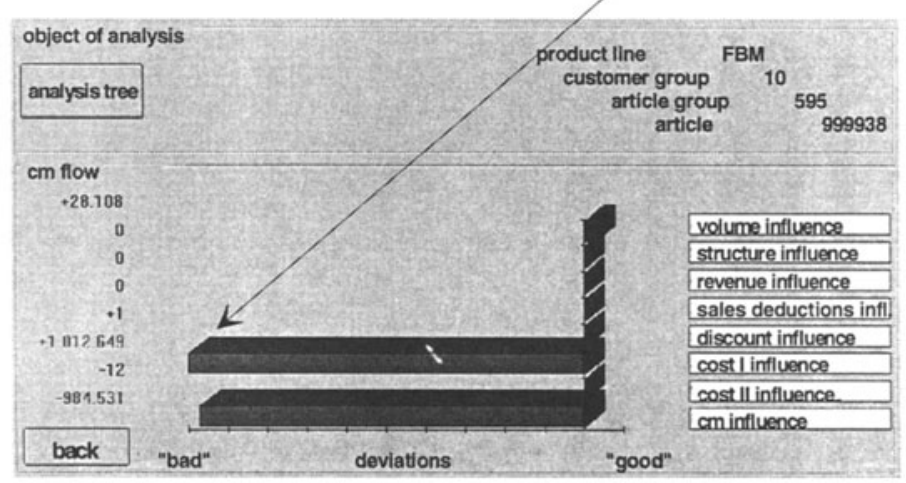

Figure 11 Case Study

Incidentally in the meantime a FORWISS project was to integrate a large part of the topdown navigation concept into the module CO (Controlling) of the SAP-System R/3. The functionality will be available in the latest release 3.0. 


\section{System Description: SIMULEX}

SIMULEX will just be mentioned in this context since - within our framework (Figure 2) - it is an example for an MIS that has some capabilities to recommend an action and to forecast its impact. So we make some progress on the path from symptoms to diagnosis, therapy, and prognosis. If the production control detects a disturbance (symptom), because a bottleneck machine broke down (diagnosis), our system simulates a large number of rescheduling strategies. SIMULEX has an experimental design module to configure a reasonable set of simulation runs. By analyzing the vast simulation output using special statistical procedures the system deducts some recommendations, e.g.: "If you split the orders $A, D$, and $H$ and give the highest priority to order $B$ you'll come back to the original schedule on day 144. The additional operating costs are 12500 DM." SIMULEX will rank the recommended policies according to a goal which is based on the turnpike scheduling philosophy.

\section{DATA MINING SYSTEMS}

A totally different approach to analyze accounting data unlike the drill-down heuristic of BETREX works bottom-up. The non-aggregated operating result records are grouped into clusters which are described by their characteristic criteria and key figures. This tool called CLUSMIN is an application of Knowledge Discovery in Databases (KDD) also known as Data Mining. The goal of KDD is the nontrivial extraction of implicit, previously unknown, and potentially useful information from data (Frawley, 1991; Bissantz, 1993; Hagedorn, 1994). To understand the notion of this research field, it is helpful to view the operating result items as points in a multidimensional search space (Figure 12). The dimensions forming the space refer to the criteria and key figures inherent in the profit data. Thus, every single record can be considered a point in space.

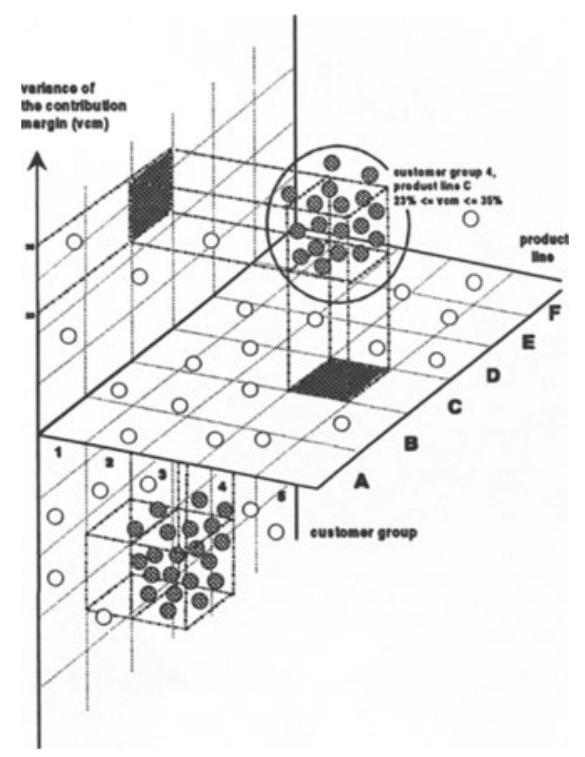

Figure 12 Clusters of operating results records 
As can be seen in Figure 12, obvious accumulations of similar records reflect interesting regularities. The notion of similar records enables the system to search for patterns in terms of sets that are meaningful to the user, in effect focusing the search.

\section{System Description: CLUSMIN}

CLUSMIN has been implemented to search for those patterns in profit data (Figure 13).

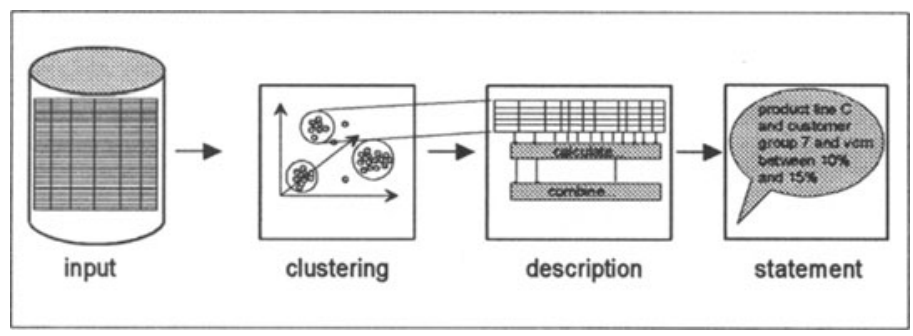

Figure 13 Architecture of CLUSMIN

The discovery procedure of CLUSMIN involves two processes: identifying useful patterns and describing them in a meaningful manner. The pattern extraction uses the statistical technique of cluster analysis to group similar records into subclasses. The ability to use statistical standard techniques is important given that the data is often inherently noisy and the patterns are therefore statistical. For our purposes, we adapted two different methods of clustering known as average linkage and sequential heuristic clustering. The existence of both numeric and non-numeric attributes in operating results data required a particular measure for calculating dissimilarity. Moreover, the clustering has to be influenced according to a user bias. This called for implementation of a module that allows the attributes to be weighed individually. Figure 14 shows the user interface.

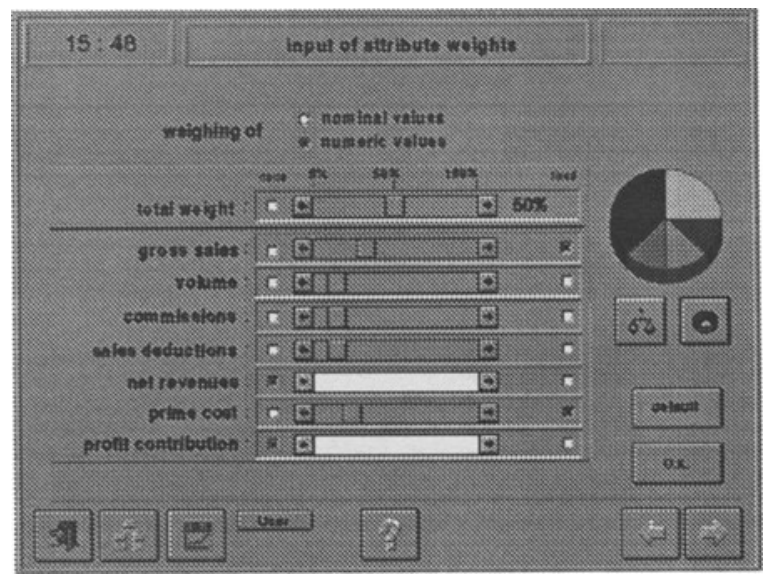

Figure 14 CLUSMIN screen for attribute weighing

Once identified, the groupings have to be described, rather than simply enumerating them. Our procedures for pattern description use a heuristic model based on different ratios which 
reflect the dispersion of attributes. To put it in simple terms, the algorithm selects those key figures and attributes for description that are statistically far less dispersed within the grouping than within the complete data. With systems like CLUSMIN, there is always the danger that the generated rules are of a trivial nature. So we are looking for ideas to suppress such statements, but up to now we did not find adequate solutions for this rather difficult problem.

\section{Case Study}

CLUSMIN was employed to analyze SAP data of a pharmaceutical company.

The system for example detected autonomously that the product groups 595 and 598 were often sold with no sales deduction at all. Please note that such a rule does not necessarily represent all sales of this product group. We look at those rules generated by CLUSMIN as an electronic suspicion. The controller him- or herself has to decide whether this suspicion is worthwhile additional analysis or not. In this example, we decided it would be worthwhile and used a graphical representation to see, if the suspicion is due to a more or less important exception or if the suspicion holds for the product group in total. We found that the detected rule was global and therefore, all the more interesting.

As well the system found some "organizational" lacks. The company had made some mistakes in the maintenance of the complicated tables of the SAP system. So we regard our data mining system as an "instrument to control the controlling". The pharmaceutical company has decided to implement CLUSMIN and transfer it from prototype to a running system.

\section{CRITIQUING SYSTEMS (MAN > MACHINE)}

In opposite to the critiquing systems that we discussed earlier in this chapter we now deal with a technique where instead of a computer criticizing a person, the person influences the future behavior of the computer. As an example we mention adaptive SDI-Systems (Selective Dissemination of Information), like they were implemented in the IBM Technical Retrieval Center (ITIRC) already in the 1970 s.

These systems have the task to keep the researchers and developers informed and to "pilot" them through the vast number of scientific publications. The interested user creates a "permanent profile of information needs" by some key words. The SDI-System checks new documents, chooses supposedly interesting documents and shows them on the display of the researcher's computer. In the case of adaptive SDI-Systems the user returns his or her satisfaction or dissatisfaction by judging the document's value. The application adapts the information profile, especially by modifying the weight of the descriptors.

Systems based on intelligent agents may offer a considerable potential to refine these techniques, e.g. when surfing in the Internet.

\section{AUTOMATIC REPORTING SYSTEMS}

At the end of the business process that derives management information stands the "finished product", in our case the presentation. A quite realistic vision is the fully automatic presentation that is transported via net.

\section{System Description: COBRAS}

At this point we turn back to the system COBRAS that is designed as an intelligent analysis and presentation software for retail audit data with a focus on automation in the client service. 
Our aim is to simplify the preparation of presentations. We collected the existing design ideas and tried to capture the expertise of the market researchers and stored that knowledge in the information system. Every market researcher has access to the GfK presentation knowhow, thus ensuring a high service quality. The system consists of the basic components shown in Figure 15.

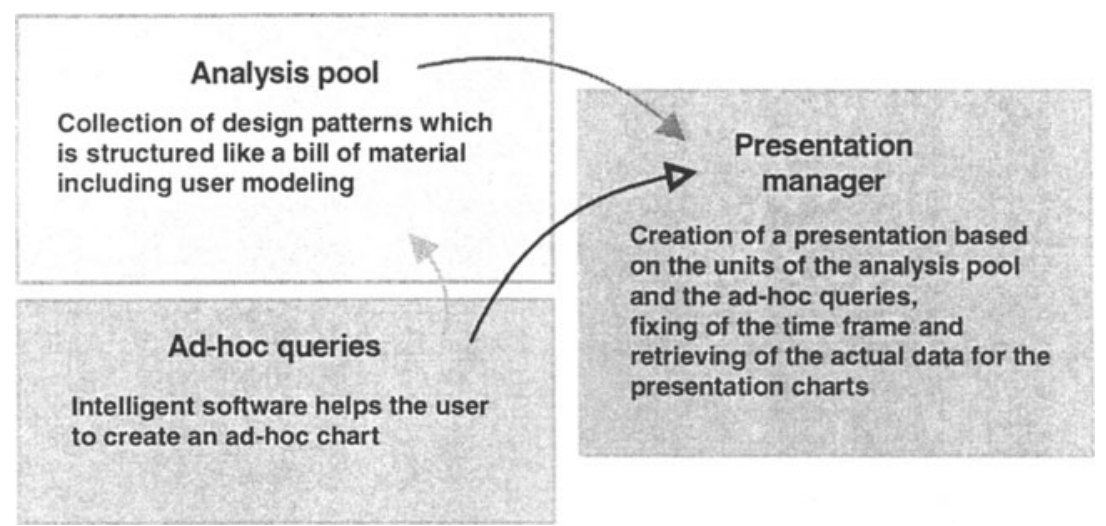

Figure 15 Basic components of COBRAS

The analysis pool represents the design patterns that were collected during the knowledge acquisition. The smallest logical unit of the analysis pool is a single chart. It consists of a diagram and/or a table, some context information, the GfK logo, copyright, and further elements. A chart shows information about a specific subject, for example the model concentration curve of a single brand (Figure 16).

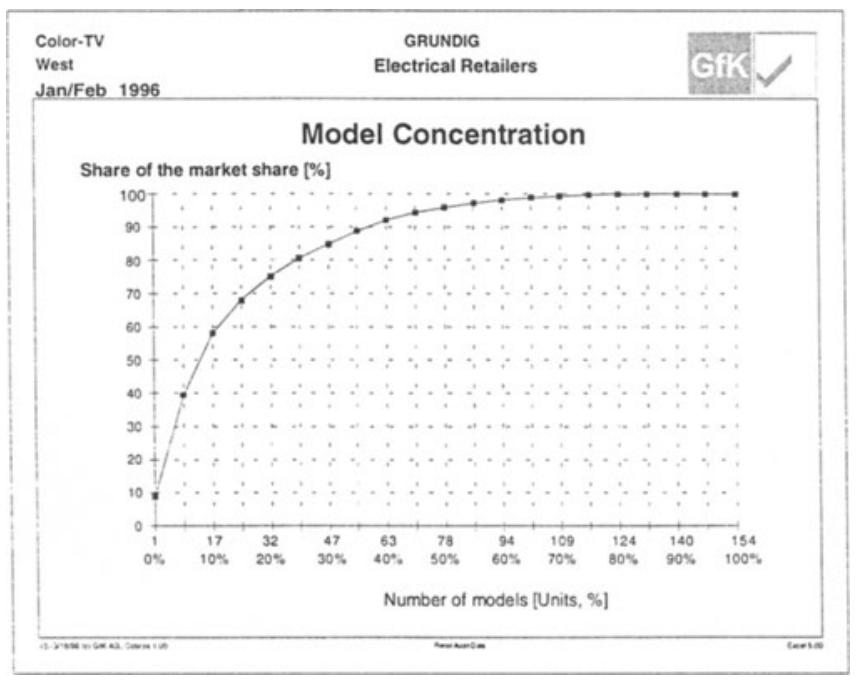

Figure 16 Model concentration 
Usually several charts belong together: the first one explains the underlying basics, another one shows the analysis results and yet another one draws conclusions or summarizes the matter. They are arranged in frameworks like the position analysis of brands in a market. The user of the system may change these analyses and save them as standards that are valid for special product groups or clients. So the GfK standard can serve as an extensive chart catalogue that also allows individual presentation profiles. Several retrieval methods enable the user to easily handle the large amount of charts in the catalogue.

Another possibility to access the database of COBRAS is to generate and process ad-hoc queries. The system offers different views on the data depending on the user's preferences (professional or inexperienced user). The design rule base again helps to choose a suitable business graphic type that is generated by the output module.

The user only interacts with the Presentation manager that helps to arrange the charts of the upper components in form of a hierarchical structured presentation. The system saves the chart patterns as well as the matching database queries, so that the user is able to "refill" an automatically generated presentation structure with the actual data in a new reporting period.

\section{Process of the Presentation Workout}

If the user wants to create a new presentation, he or she chooses several suitable analysis concepts and structures the presentation hierarchically (Figure 17,1). This phase can start before the new data is available. Mostly the client's questions are known before, such as: "How well was our new product distributed?" The Presentation manager offers the possibility to assign time frames to analyses so that the presentation can be scheduled.

Using descriptors, such as Product, Place, Price, or Promotion, the system generates a ranking of the suitable charts (Figure 17,2). The degree of a chart's suitability is measured by the fitting of the given descriptors as well as the occasion, such as a presentation for the board of directors. Now the user may choose some of the proposed charts, their order and the underlying data.

Until step 2 the system does not need the actual data. In the following phase the system retrieves the data and checks the necessary presentation time (Figure 17, 3). Then the user may cancel some charts or add new ones (Figure 17, 4).

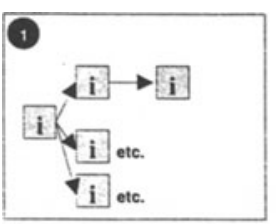

Determining the goal, creating problem-oriented analysis concepts. assigning time frames to analysis units

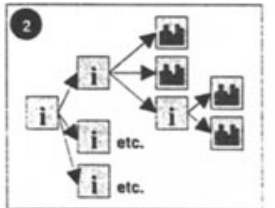

Choosing the most suitable charts, fixing the market segment and ordering the charts

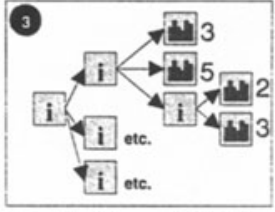

Selecting the needed data, calculating corresponding presentation time

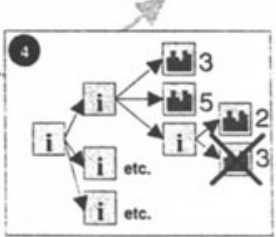

Depending on the given time frame adding or removing charts

Figure 17 Process of the presentation configuration 


\section{Automatic Analysis of Financial Statements}

Our last example leads us to the automatically generated report on the left-hand side of the scale (Figure 2). In connection with that we would like to touch user modeling. In the field of business administration the term addressee modeling might be more appropriate because the reports often are addressed to institutions rather than to persons (see below). The existing work of Theoretical Computer Science in this field is respectable, but - as far as we can see there are not many actual implementations. We think that it is the job of the Information Systems research community to close this gap.

In order to be able to measure the economic success of a business as an outsider, one only has access to information contained in public financial records. This data is mainly drawn from the balance sheet and the profit and loss statement.

Furthermore it is important to realize how other external partners (such as banks, shareholders, etc.) view the business. The creditor's decision is mainly affected by the results of a balance sheet analysis (Hamm, 1994, p.132). The company's desire to continuously know its credit rating and financial standing therefore demands a continuous financial analysis.

\section{System Description: UNTERNEHMENSREPORT}

The knowledge-based system described here, UNTERNEHMENSREPORT II (Haase, 1995), was created in cooperation with FORWISS and DATEV e.G., Nuremberg, a large German cooperative association. This association supports German tax consultants with information technologies. The typical clients of the tax consultants are small to medium-sized companies. The existing (running) system UNTERNEHMENSREPORT I which is able to analyze only two consecutive years is used in some 14000 tax consultants' offices. This system generates an expertise of 20 to 30 pages referring to a company's economic performance. Table 1 gives an output example (translated into English, DATEV, 1992, p. 8).

Table 1 Output example of UNTERNEHMENSREPORT I

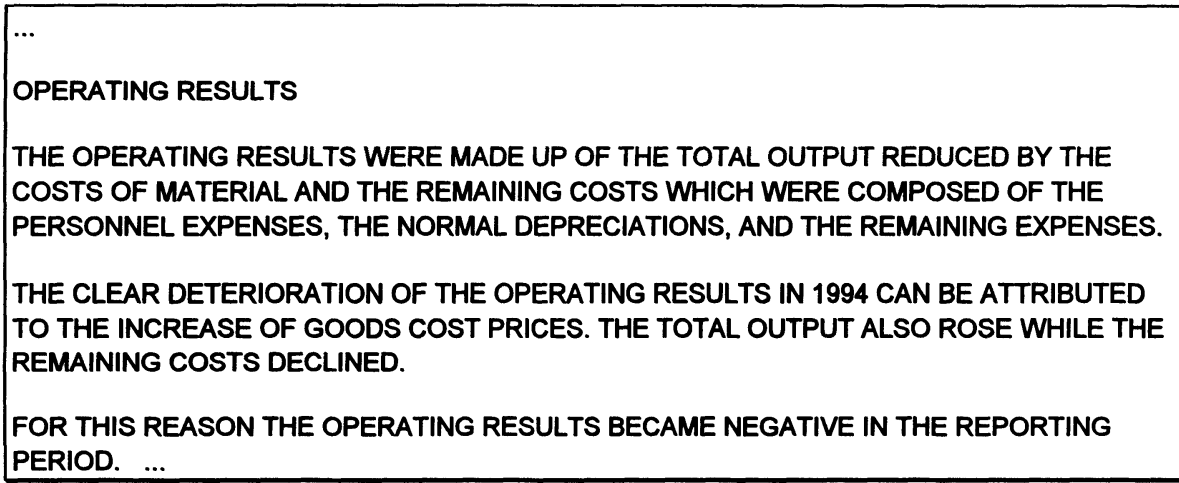

UNTERNEHMENSREPORT II will offer a lot of additional features. It examines time series on a yearly basis from two to ten consecutive years and on a monthly basis up to 24 months. Moreover, the application analyzes the individual client's industry averages and data which is projected up to five years into the future. It is left up to the tax consultant to choose the time horizons. At DATEV average ratios are available for a substantial number of industries. The system is refined in a way to even distinguish differences between very small, small and medium-sized companies within a single industry. 
Figure 18 offers an overview of the dimensions that are integrated into the analysis of UNTERNEHMENSREPORT II (the analysis models will be described below).

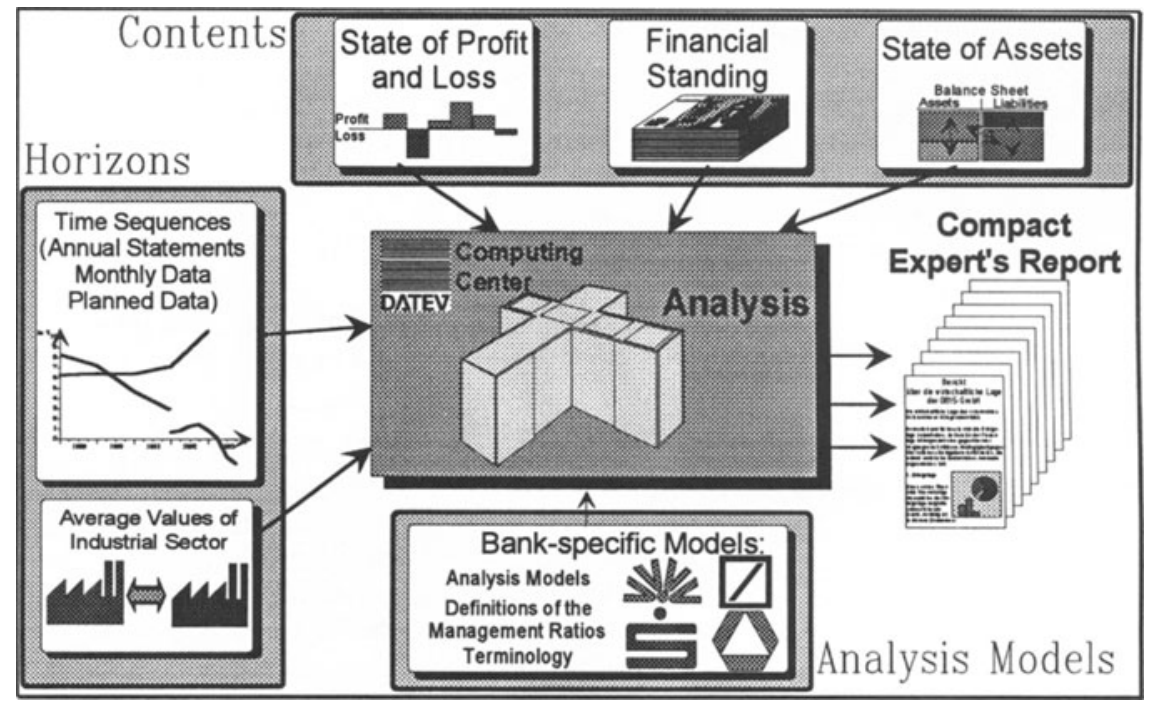

Figure 18 Integrated dimensions in UNTERNEHMENSREPORT II

\section{Addressee-Orientation}

An important factor enabling a system to produce high-quality reports automatically is to employ models about the addressee. The report may be for the tax consultant who could use it as a basis for a discussion with his or her client. If the consultants want to give the expertise directly and unchanged to their clients, then the addressee may be the entrepreneur or a manager. A potential external recipient is the company's house bank, for example to apply for a credit.

Depending on the addressee, the user can select whether the report should contain tables or not and whether it will include diagrams and if so, which kind of graphical representation should be preferred.

Especially if the report is intended for a top manager of the client's organization, recommendations how to react to a specific situation may be useful. It is not advisable to use evaluations and risky formulations for all addressees (especially the external ones). The recommendation and judgment problem was solved by providing the respective pieces of text with a special attribute. The default choice for a report is expertises without these marked passages.

Another option is the possibility to choose the level of detail of the expertise. For this reason we implemented a hierarchical design for the expert system. It consists of different layers. The higher the layer, the more consolidated the included information. The top layer, for example, produces text concerning the supreme goal of the analysis, the economic situation of the company. So UNTERNEHMENSREPORT II is able to produce an expertise that only contains the levels of consolidation down to a user-specified layer (Figure 19). The standard report will include the upper four layers (of five). A brief executive summary will be cut off between 
layer two and three. The long version of the report with all its details, for example for the book-keeper's assistant, consists of all layers.

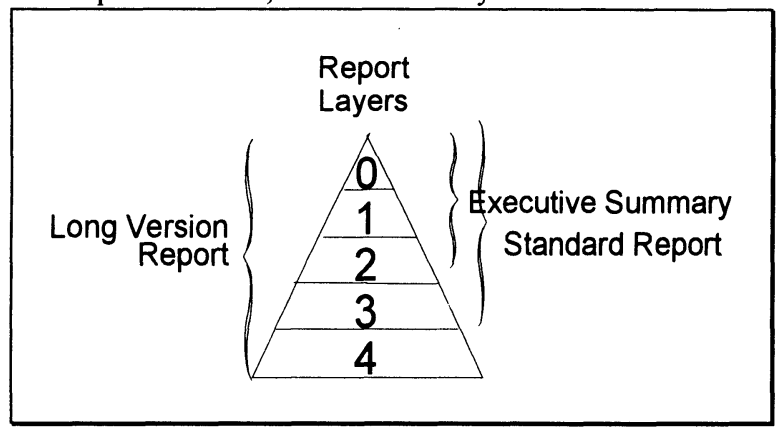

Figure 19 Level of detail

If the addressee of the expertise is a bank, then it is important to follow the bank's guidelines of carrying out credit investigations. Those guidelines partly vary with regard to the definitions of ratios and business terms. That leads to difficulties with the so-called surface text-generation that deals with syntax problems and lexical choice (Rankin, 1993). The German language creates a range of problems here that are not existing in other languages like English.

The system cannot use a fixed business vocabulary, since it has to follow the internal vocabulary of the banks. It holds lists with the special terms and their gender, genitive ending syllable and more. In German, not only the indefinite and definite articles (indefinite article: masc. "ein", fem. "eine", neut. "ein", definite article: masc. "der", fem. "die", neut. "das") depend on the gender of the subject but also the pronouns and many word endings.

E.g., the term "Teilbetriebserfolg" used by the DEUTSCHE BANK for "Operating Results" has another gender than the BAYERISCHE VEREINSBANK's synonym "Betriebsergebnis", which is a neutral noun, but also has a different ending for the genitive case, where "Teilbetriebserfolg" gets the ending "s", while "Betriebsergebnis" will read "Betriebsergebnisses".

Of course, the banks are free to modify their evaluating procedure (e.g. the ratios) without regarding the consequences for UNTERNEHMENSREPORT II. To deal with these modifications we separated this part ot the knowledge base and created special lists. So the knowledge structure is independent of the bank's decisions. Those separated lists contain information concerning the algorithms to compute the ratios and the reliability of the resulting figures. Following Davis and Buchanan we might call that "meta-level knowledge" (Davis and Buchanan, 1984).

\section{CONCLUSION}

A higher degree of automation in management information assumes that the system knows the user very well. To become more general we want to pick up Negroponte's thesis, saying we are just converting from the information society into the post-information society. The latter is characterized by a turn away from mass communication towards individual communication.

This is one reason why we should pay much attention to Active MIS including user or addressee modeling. 


\section{REFERENCES}

Beys, O. (1994) PANELYSER - Ein Beitrag zur effizienten Analyse von Paneldaten mit Hilfe wissensbasierter Elemente. $\mathrm{PhD}$ thesis, Nürnberg.

Beys, O. and Müller, S. (1992) Analysis of Retail Audit Data with Expertise Systems, in Do New Technologies Help or Hinder Marketing Decisions? (ed. ESOMAR), Proceedings of the ESOMAR Conference, Rotterdam, 113-123.

Beys, O., Fischer, M., Tripmaker, S., and Mertens, P. (1992) Wissenbasierte Analyse von Handelspaneldaten. Marketing ZFP, 3(14), 157-166.

Bissantz, N. and Hagedorn, J. (1993) Data Mining (Datenmustererkennung). Wirtschaftsinformatik, 5(35), 481-487.

Bissantz, N., Hagedorn, J., and Mertens, P. (1995) Top-down Navigation and Knowledge Discovery in SAP Operating Results Data: The BETREX System, in Managing Information \& Communications in a Changing Global Environment (ed. M. Khosrowpour), Proceedings of the 1995 Information Resources Management Association International Conference, May 21-24th, Atlanta, Georgia USA, 420.

DATEV e.G. (ed.) (1992) UNTERNEHMENSREPORT - Produktinformation mit Anwendungsbeispiel. Nürnberg.

Davis, R. and Buchanan, B.G. (1984) Meta-Level Knowledge, in Rule-Based Expert Systems (ed. B.G. Buchanan and E.H. Shortliffe), The MYCIN Experiments of the Standford Heuristic Programming Project, Reading, Massachusetts, 507-530.

Frawley, J.F., Piatetsky-Shapiro, G., and Matheus, C.J. (1991) Knowledge Discovery in Databases: An Overview. AI Magazine, 3(13), 57-70.

Haase, M. (1995) Wissensbasierte Jahresabschlußanalyse mit Unternehmensreport. Theorie und Praxis der Wirtschaftsinformatik HMD, 182, 37-44.

Mertens, P. (1992) An Expert System for Analyzing the Profit and Financial Situation of Small and Medium-Sized Companies, in Proceedings on World Congress on Expert Systems, Orlando, 1269-1276.

Mertens, P. (1989) Derivation of Verbal Expertises from Accounting Data, in Expert Systems in Economics, Banking, and Management (ed. L.F. Pau, J. Motiwalla, Y.H. Pao, and H.H. Peh), Amsterdam, NewYork, Oxford, Tokyo, 341-350.

Parfitt, J.H. and Collins, B.J.K. (1968) Use of Consumer Panels for Brand-Share Prediction. Journal of Marketing Research, 5, 131-145.

Rankin, I. (1993) Natural Language Generation in Critiquing. The Knowledge Engineering Review, 4, 329-347.

Stonebraker, M., Agrawal, R., Dayal, U., Neuhold, E.J., and Reuter, A. (1993) DBMS Research at a Crossroads: The Vienna Update, in Proceedings of the 19th VLDB Conference (ed. R. Agrawal, S. Baker, and D. Bell), Dublin, 701. 


\section{BIOGRAPHY}

Prof. Dr. Dr. h. c. mult. Peter Mertens

Peter Mertens, born 1937, is director of the Department for Information Systems Research at the University of Erlangen-Nuremberg, Germany. He is the deputy-spokesperson of the executive committee at the Bavarian Research Center for Knowledge-Based Systems (FORWISS). He is also the editor-in-chief of WIRTSCHAFTSINFORMATIK, the leading German journal dedicated to Information Systems Research.

Professor Mertens graduated from the Technical University of Darmstadt, Germany with a degree in industrial engineering. He worked at several universities, before joining a major IT-consulting firm, at first in a management position, later as managing director.

His main research interests are: IS in marketing, production, logistics, and controlling; MIS; Expert Systems; Data Mining; Workflow Management Systems; Industry-specific application architectures; Virtual enterprises.

Dipl.-Inf. Jürgen Hagedorn, Dipl.-Kfm. Margit Fischer, Dipl.-Kfm. Nicolas Bissantz, and Dipl.-Kfm. Michael Haase

They all belong to Mertens' research staff at the Group for Information Systems Research of FORWISS. 